\title{
Theoretical Study on Thermal Release of Helium-3 in Lunar Ilmenite
}

\author{
Hongqing Song ${ }^{1, *}$, Jie Zhang ${ }^{1}$, Yueqiang Sun ${ }^{2}$, Yongping Li ${ }^{2}$, Xianguo Zhang ${ }^{2}$, Dongyu Ma ${ }^{1}$ and Jue Kou ${ }^{1}$ \\ 1 School of Civil and Resource Engineering, University of Science and Technology Beijing, \\ Beijing 100083, China; zj39kevin@163.com (J.Z.); mady18810778706@163.com (D.M.); \\ koujue@ustb.edu.cn (J.K.) \\ 2 National Space Science Center, Chinese Academy of Science, Beijing 100190, China; syq@nssc.ac.cn (Y.S.); \\ lyp@nssc.ac.cn (Y.L.); zhangxg@nssc.ac.cn (X.Z.) \\ * Correspondence: songhongqing@ustb.edu.cn; Tel.: +86-010-82376239
}

Citation: Song, H.; Zhang, J.; Sun, Y.; Li, Y.; Zhang, X.; Ma, D.; Kou, J.

Theoretical Study on Thermal Release of Helium-3 in Lunar Ilmenite. Minerals 2021, 11, 319. https:// doi.org/10.3390/min11030319

Academic Editors: Kattathu J. Mathew and Yann Morizet

Received: 28 January 2021

Accepted: 17 March 2021

Published: 19 March 2021

Publisher's Note: MDPI stays neutral with regard to jurisdictional claims in published maps and institutional affiliations.

Copyright: (C) 2021 by the authors. Licensee MDPI, Basel, Switzerland. This article is an open access article distributed under the terms and conditions of the Creative Commons Attribution (CC BY) license (https:// creativecommons.org/licenses/by/ $4.0 /)$.
Abstract: The in-situ utilization of lunar helium-3 resource is crucial to manned lunar landings and lunar base construction. Ilmenite was selected as the representative mineral which preserves most of the helium-3 in lunar soil. The implantation of helium-3 ions into ilmenite was simulated to figure out the concentration profile of helium-3 trapped in lunar ilmenite. Based on the obtained concentration profile, the thermal release model for molecular dynamics was established to investigate the diffusion and release of helium-3 in ilmenite. The optimal heating temperature, the diffusion coefficient, and the release rate of helium-3 were analyzed. The heating time of helium-3 in lunar ilmenite under actual lunar conditions was also studied using similitude analysis. The results show that after the implantation of helium-3 into lunar ilmenite, it is mainly trapped in vacancies and interstitials of ilmenite crystal and the corresponding concentration profile follows a Gaussian distribution. As the heating temperature rises, the cumulative amounts of released helium-3 increase rapidly at first and then tend to stabilize. The optimal heating temperature of helium- 3 is about $1000 \mathrm{~K}$ and the corresponding cumulative release amount is about $74 \%$. The diffusion coefficient and activation energy of helium-3 increase with the temperature. When the energy of helium-3 is higher than the binding energy of the ilmenite lattice, the helium-3 is released rapidly on the microscale. Furthermore, when the heating temperature increases, the heating time for thermal release of helium- 3 under actual lunar conditions decreases. For the optimal heating temperature of $1000 \mathrm{~K}$, the thermal release time of helium-3 is about $1 \mathrm{~s}$. The research could provide a theoretical basis for in-situ helium-3 resources utilization on the moon.

Keywords: helium-3 resources; in-situ resources utilization; thermal release; lunar ilmenite; heating temperature

\section{Introduction}

Lunar soil, as the most accessible and widely distributed material on the Moon, has high scientific, engineering, and economic value, especially in the field of in-situ utilization of lunar resources [1,2]. The minerals contained in the lunar soil include ilmenite, olivine, plagioclase, pyroxene, and so on $[1,3]$. These minerals can be used as building materials for lunar bases after beneficiation and processing [4].

There is also a large amount of volatiles trapped in the lunar soil, including water ice, helium-3, hydrogen, and so on [5-8]. Many studies have focused on the diffusion of these volatiles on the lunar surface [9-11]. In addition, it is also of great significance to realize the in-situ mining and utilization of the volatile resources. For example, previous exploration and research have shown that a large amount of water ice existed in permanently shadowed regions of the lunar south pole [6,7]. The water ice can be obtained directly on the Moon via an in-situ thermal mining method [12]. After the purification, the obtained water ice can be electrolyzed to produce hydrogen and oxygen, which are stored in the forms of liquid 
hydrogen and liquid oxygen, respectively [13]. Liquid hydrogen can be used as fuel for spacecraft, and liquid oxygen can be used for breathing of the crew on the lunar base [14].

Helium-3 is a clean fuel for controllable nuclear fusion $[15,16]$. The electricity can be generated through nuclear fusion reactions with $\mathrm{D}$ and ${ }^{3} \mathrm{He}$ as the fuel, which can provide energy for the establishment and operation of a lunar base and a solution for the alleviation of the energy crisis on Earth. Compared to Earth, helium-3 is easier to accumulate on the surface of the Moon [17]. The helium-3 trapped in lunar soil mainly came from the solar wind, which mainly consists of protons and electrons [18]. The solar wind also contains rare gas isotopes such as ${ }^{3} \mathrm{He},{ }^{4} \mathrm{He},{ }^{36} \mathrm{Ar}$, and ${ }^{40} \mathrm{Ar}$ [19]. Since the Moon has almost no atmosphere and magnetic field, the solar wind can continue to bombard the surface of the Moon without being deflected or attenuated, which allows the lunar soil to capture a large amount of helium-3. It is estimated that the amount of helium-3 on the lunar surface is up to $6.50 \times 10^{8} \mathrm{~kg}$ [15].

Different lunar soil minerals have different abilities to accumulate helium-3. Previous studies have shown that it was easier for ilmenite to trap helium-3 than for other lunar soil minerals such as olivine and plagioclase $[20,21]$. The crystal of ilmenite has a hexagonal close-packed structure, and the lattice spacing of ilmenite is also similar to the size of helium-3 atoms. Therefore, compared to other minerals, the lattice structure of ilmenite is more suitable to trap helium-3 atoms. Since the composition and amounts of lunar soil minerals are different in different lunar regions, especially the ilmenite distribution is non-uniform on the lunar surface, the distribution of helium-3 also shows great differences. For example, compared with the Mare Serenitatis region of the Moon, which is dominated by low and medium titanium basalts (or low ilmenite abundance), the Mare Tranquillitatis region dominated by high titanium basalts (or high ilmenite abundance) contains more helium-3 resources [22-24].

Studying the forms and thermal release characteristics of helium-3 in lunar soil is of great significance for in-situ thermal mining of helium-3 [25-29]. Previous studies have shown that there were many forms of helium-3 in lunar soil [30]. Due to the bombardment of energetic particles in solar wind on lunar soil minerals, the helium-3 atoms existed mainly in lattice interstitials and vacancies of lunar soil minerals [30]. In addition, different forms of helium-3 also affect the diffusion of helium-3 in lunar soil minerals, thereby affecting its thermal release characteristics. Anufriev investigated the diffusion of helium isotopes in lunar soil samples from Luna-24 and found that due to the radiation damage, the diffusion of helium isotopes in lunar soil did not obey Fick's law. Helium atoms were bound in the damaged mineral crystals, and extra energy was required to cause the displacement of helium-3 atoms [31]. Harris-Kuhlman et al. studied the forms and diffusion of helium in simulated lunar soil, which was represented by ilmenite. Helium-3 and helium-4 were implanted into the simulated lunar soil with different energies and doses via plasma source ion implantation (PSII). It was found that four different activation energies existed during diffusion of helium-3 and helium-4, which corresponded to the detrapping from oxygen and constitutional vacancies and diffusion through two amorphous layers, respectively [32]. Futagami et al. selected ilmenite and olivine to represent the simulated lunar soil. Combined with ion implantation experiments, they studied the thermal release process of volatiles such as helium in simulated lunar soil. They found that the thermal release temperature of volatiles in two minerals depended on the energy and dose of incident ions [33]. Srinivasan et al. investigated the thermal release characteristics of noble gases ( $\mathrm{He}, \mathrm{Ne}, \mathrm{Ar}, \mathrm{Kr}, \mathrm{Xe})$ in Apollo 15 samples with a stepwise heating method and found that compared with heavy noble gases the light noble gases $(\mathrm{He}, \mathrm{Ne})$ were released at lower temperatures and the majority of them were released below $700{ }^{\circ} \mathrm{C}$ [26].

When studying the thermal release characteristics of helium-3, it is not only necessary to consider the forms and diffusion of helium-3 atoms in minerals, but also the concentration profile along the implantation depth in lunar soil minerals. Gas ion probe analysis shows that the concentration profile of solar wind injected gas in lunar soil minerals presented a shape of Gaussian distribution, which also varied with types of lunar soil minerals. 
For example, the concentration profile of helium-3 in ilmenite and plagioclase was unimodal, while the concentration profile in olivine was bimodal $[34,35]$. The concentration profiles of helium-3 in lunar soil minerals affect the diffusion of helium-3 and its thermal release. However, related research is currently lacking, especially microscopic studies on the influence of the concentration profile of helium- 3 on the diffusion process, and the corresponding thermal release mechanism.

In this paper, the thermal release process of helium-3 was investigated in ilmenite, due to its higher ability to trap helium-3 compared with other lunar soil minerals. At first, the concentration profile of helium-3 trapped in ilmenite was investigated using numerical simulations using SRIM (Stopping and Range of Ions in Matter) code. Then, the molecular dynamics simulations for thermal release of helium-3 were performed to analyze the diffusion and release of helium-3 in ilmenite on the microscale. Finally, the heating time of helium-3 in ilmenite under actual lunar conditions was studied using similitude analysis.

\section{Concentration Profile of Helium-3 Trapped in Lunar Ilmenite}

The implantation of helium-3 ions into the lunar ilmenite includes the impacting of ions on a solid surface and ions migration in the solid. In this work, SRIM-2013 software is utilized to calculate the range and damages of implanted ions in a target [36]. It can simulate the implantation process of helium-3 ions into the lunar ilmenite.

\subsection{Theoretical Model about Implantation of Helium-3 into Ilmenite}

Binary collision theory is applied between incident helium-3 ions and target ilmenite atoms. Meanwhile, the motion of incident ions after the implantation is tracked using the Monte Carlo method, and the particle position, energy loss, and other parameters of target atoms are recorded. Then, the average values and statistical errors for different output parameters are calculated [36].

There are two collision ways between incident ions and target atoms, including the elastic collision between ions and nuclei of target atoms and inelastic collision between ions and electrons of target atoms. For the collision between helium-3 ions and ilmenite atoms, the energy loss of helium-3 ions per unit length is expressed as Equation (1):

$$
S=\left(\frac{d E}{d x}\right)_{n}+\left(\frac{d E}{d x}\right)_{e}
$$

where $\left(\frac{d E}{d x}\right)_{n}$ is the energy loss for the collision between helium-3 ions and nuclei of target ilmenite atoms, with unit $\mathrm{keV} / \AA ;\left(\frac{d E}{d x}\right)_{e}$ is the energy loss for the collision between helium-3 ions and electrons of target ilmenite atoms, with unit $\mathrm{keV} / \AA$. The inelastic electron collision is the main energy loss for the implantation of helium-3 ions into the ilmenite atoms.

Assuming that the incident direction of helium-3 ions is perpendicular to the surface of ilmenite, the concentration distribution with implantation depth is shown in Equation (2):

$$
N(x)=\frac{D}{\sqrt{2 \pi} \cdot \Delta R_{p}} \cdot e^{-\frac{\left(x-R_{p}\right)^{2}}{2 \Delta R_{p}^{2}}}
$$

where $x$ is the depth of implanted helium-3 ions, with unit $\AA$; $N(x)$ is the amount of implanted helium-3 ions per unit volume at depth $x$, with unit $1 / \AA^{3} ; D$ is the dose of incident helium- 3 ions, with unit ions $/ \AA^{2} ; R_{p}$ is the mean projected range of all ions on the $x$ axis, with unit $\AA ; \Delta R_{p}$ is the corresponding standard error, with unit $\AA$.

The energy transfer of incident helium-3 ions is shown in Equation (3) [37]:

$$
E_{i}^{0}=E_{i}^{p}+E_{r}^{p}+E_{i}^{I}+E_{r}^{I}
$$


where $E_{i}{ }^{0}$ is the energy of incident ions, with unit keV; $E_{i}{ }^{p}$ is the energy loss of incident ions into phonons in target atoms, with unit $\mathrm{keV} ; E_{r}{ }^{p}$ is the energy loss of recoiling atoms into phonons in target atoms, with unit $\mathrm{keV} ; E_{i}{ }^{I}$ is the ionization energy loss of incident ions, with unit keV; $E_{r}{ }^{I}$ is the ionization energy loss of recoiling atoms, with unit keV. In the simulation, the target layer is divided into 100 cells with equal width. The number of vacancies, the phonon energy in the lattice, the ionization energy loss, and the energy transfer to recoiling atoms are calculated in each cell, respectively.

\subsection{Concentration Profile of Implanted Helium-3 Ions in Lunar Ilmenite}

The average speed of solar wind on the surface of the Moon is about $468 \mathrm{~km} / \mathrm{s}$ and the average energy is about $1 \mathrm{keV} / \mathrm{amu}$ [38], and the average energy of helium ions in the solar wind is about $4 \mathrm{keV}$. The diffusion of helium-3 implanted from the solar wind in actual lunar soil could take a long time to reach equilibrium, which led to greater depth on the concentration profile of helium-3 in lunar soil grains compared with the implantation depth of solar wind ions [39]. However, the long-term diffusion process of helium-3 after implantation into ilmenite cannot be characterized via the SRIM simulation. Therefore, the incident energy of helium-3 is increased in this work in order to obtain a similar concentration profile of helium-3 with that in actual lunar soil grains, and the energy of incident helium-3 ions is selected as $50 \mathrm{keV}$ [40]. The dose of incident helium-3 ions is $4 \times 10^{13}$ ions $/ \mathrm{cm}^{2}$ and the density of ilmenite is $4.7 \mathrm{~g} / \mathrm{cm}^{3}$. Other parameters required for the simulation of implantation of helium-3 into ilmenite are listed in Table 1. The direction of incident helium-3 ions is perpendicular to the target ilmenite surface.

Table 1. Parameters required for SRIM (Stopping and Range of Ions in Matter) simulation of helium-3 implantation into ilmenite.

\begin{tabular}{cccccc}
\hline \multicolumn{2}{c}{ Parameters of Incident Ions } & \multicolumn{3}{c}{ Parameters of Target Atoms } \\
\hline \multirow{2}{*}{ Species } & Energy(keV) & Dose $\left(\mathbf{i o n s} / \mathbf{c m}^{\mathbf{2}}\right)$ & Element & Stoichiometry & Density $\left(\mathbf{g} / \mathbf{c m}^{3}\right)$ \\
\hline \multirow{3}{*}{ Helium-3 } & \multirow{2}{*}{50} & \multirow{2}{*}{$4 \times 10^{13}$} & $\mathrm{Fe}$ & 1 & \\
& & & $\mathrm{Ti}$ & 1 & 4.7 \\
& & & $\mathrm{O}$ & 3 & \\
\hline
\end{tabular}

Figure 1 shows the concentration profile of implanted helium-3 ions in lunar ilmenite. It is seen that the concentration profile of implanted helium-3 ions in lunar ilmenite presented the shape of Gaussian distribution. The peak appeared at about $245 \mathrm{~nm}$. The maximum implantation depth was about $423 \mathrm{~nm}$, which was in the same order of magnitude as the range of concentration profile of helium-3 ions in actual lunar ilmenite from Apollo samples [34]. In addition, every collision between incident helium-3 ions and the target ilmenite atoms produced a lattice vacancy, as indicated in red dots in the inset of Figure 1. The impacted target atoms continued to impact other atoms, resulting in more vacancies (white dots in the inset of Figure 1).

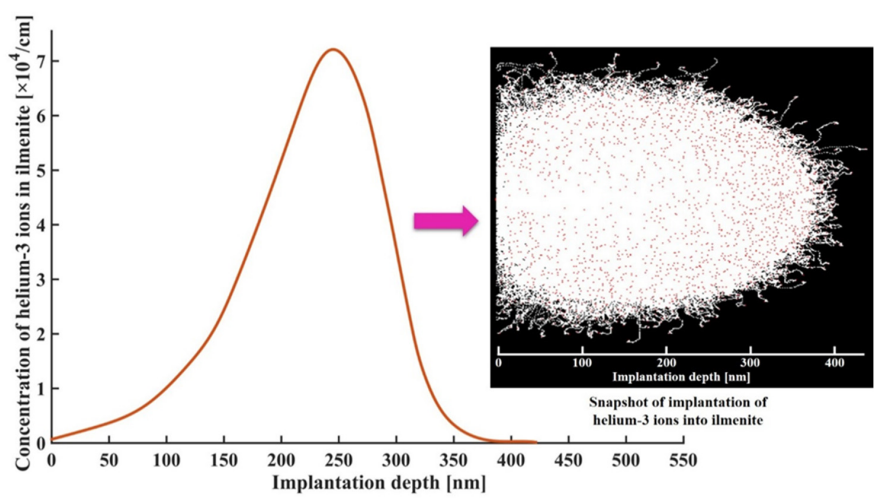

Figure 1. Concentration profile of implanted helium-3 ions in lunar ilmenite. The inset shows a snapshot of the implantation of helium-3 ions into ilmenite. 


\section{Thermal Release Model of Helium-3}

\subsection{Molecular Dynamics Simulations}

\subsubsection{Geometric Models for Helium-3 Thermal Release}

As shown in Figure 2, according to the obtained concentration profile of Gaussian distribution, helium-3 atoms were implanted into lattice vacancies and interstitials of lunar ilmenite for the initial configuration of the thermal release model. The dimensions of the simulation box along $x, y, z$ directions were $4.2 \mathrm{~nm}, 3 \mathrm{~nm}$, and $3 \mathrm{~nm}$, respectively. The thickness of the trapped helium-3 layer was $1 \mathrm{~nm}$, and the concentration peak appeared at about $0.55 \mathrm{~nm}$. Considering the highly vacuum condition (about $10^{-12} \mathrm{~Pa}$ ) on the lunar surface, a vacuum zone was set with a thickness of $2.1 \mathrm{~nm}$ over the helium layer to accommodate the released helium-3 atoms. The concentration of helium-3 atoms was about $3 \times 10^{22}$ ions $/ \mathrm{cm}^{3}$. Meanwhile, as the comparison, an initial configuration with a concentration profile of uniform distribution was also established under the same thickness and dose of helium-3 layer.

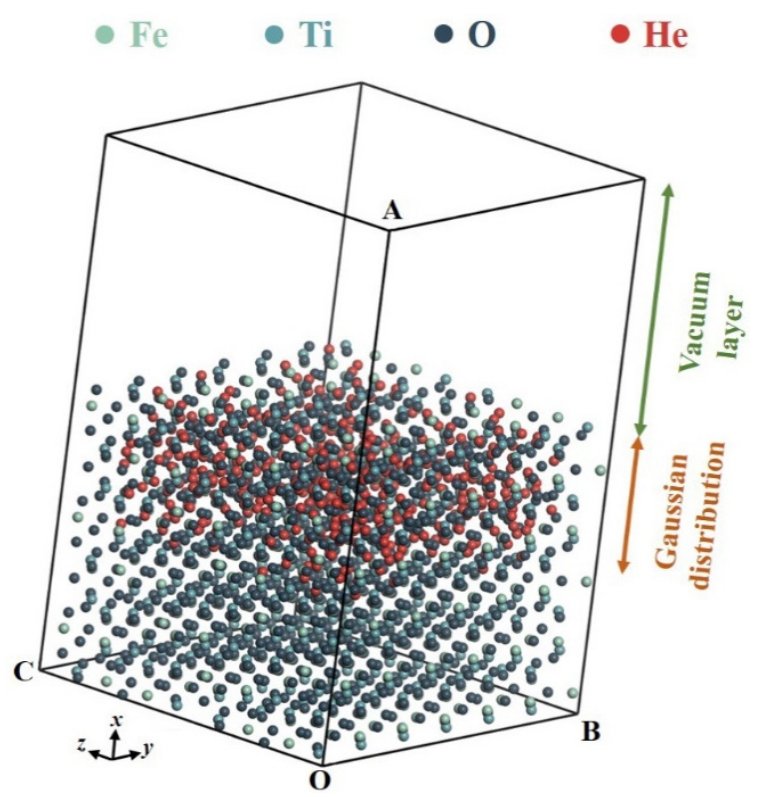

Figure 2. Geometric model of helium-3 atoms in ilmenite with Gaussian distribution for molecular dynamics simulations.

\subsubsection{Potential Models for Molecular Dynamics}

In molecular dynamics simulations, the determination of reasonable atomic potential parameters is crucial to the accuracy of simulation results. The Ti-Ti, Ti-O, O-O, and Fe-O interactions can be characterized using Born-Mayer-Huggins (BMH) potential as shown in Equation (4) [41]:

$$
U_{i j}(r)=\frac{q_{i} q_{j}}{r_{i j}}+A_{i j} \exp \left(-B_{i j} r_{i j}\right)-\frac{C_{i j}}{r_{i j}{ }^{6}}
$$

where $U_{i j}(r)$ is the $\mathrm{BMH}$ potential between atom types $i$ and $j ; q_{i}$ and $q_{j}$ are partial charges of atom types $i$ and $j$, respectively; $r_{i j}$ is the distance between atom types $i$ and $j ; A_{i j}, B_{i j}$, and $C_{i j}$ are empirical parameters. The detailed potential parameters for Ti-Ti, Ti-O, O-O, and $\mathrm{Fe}-\mathrm{O}$ interactions are shown in Table 2. 
Table 2. Potential parameters for Ti-Ti, Ti-O, O-O, and Fe-O interactions with BMH (BornMayer-Huggins) potential.

\begin{tabular}{cccccc}
\hline \multicolumn{2}{c}{ Atom Type } & $\boldsymbol{A}_{i j}(\mathbf{e V})$ & $\boldsymbol{B}_{i j}(1 / \AA)$ & $\boldsymbol{C}_{i j}\left(\mathbf{e V} \cdot \AA^{6}\right)$ & References \\
\hline $\mathrm{Ti}$ & $\mathrm{Ti}$ & $35,133.15$ & 6.25 & 0 & {$[41]$} \\
$\mathrm{Ti}$ & $\mathrm{O}$ & $242,696.25$ & 6.06 & 0 & {$[41]$} \\
$\mathrm{O}$ & $\mathrm{O}$ & $1,497,693.51$ & 5.88 & 17.35 & {$[41]$} \\
$\mathrm{Fe}$ & $\mathrm{O}$ & 1900.21 & 3.45 & 0 & {$[42]$} \\
\hline
\end{tabular}

The He-He, Fe-Fe, and Fe-Ti interactions can be characterized using Lennard-Jones (LJ) potential as shown in Equation (5) [43]:

$$
U_{i^{\prime} j^{\prime}}^{\prime}(r)=\frac{D_{i^{\prime} j^{\prime}}}{r_{i^{\prime} j^{\prime}}{ }^{12}}-\frac{E_{i^{\prime} j^{\prime}}}{r_{i^{\prime} j^{\prime}} 6}
$$

where $U_{i^{\prime} j^{\prime}}^{\prime}(r)$ is the LJ potential between atom types $i^{\prime}$ and $j^{\prime} ; r_{i^{\prime} j^{\prime}}$ is the distance between atom types $i^{\prime}$ and $j^{\prime} ; D_{i^{\prime} j^{\prime}}$ and $E_{i^{\prime} j^{\prime}}$ are empirical parameters. The detailed potential parameters for He-He, Fe-Fe, and Fe-Ti interactions are shown in Table 3.

Table 3. Potential parameters for He-He, Fe-Fe, and Fe-Ti interactions with LJ (Lennard-Jones) potential.

\begin{tabular}{|c|c|c|c|c|}
\hline \multicolumn{2}{|c|}{ Atom Type } & $D_{i^{\prime} j^{\prime}}\left(\mathrm{eV} \cdot \AA^{12}\right)$ & $E_{i^{\prime} j^{\prime}}\left(\mathrm{eV} \cdot \AA^{6}\right)$ & References \\
\hline $\mathrm{He}$ & $\mathrm{He}$ & 69.3559 & 0.493712 & [44] \\
\hline $\mathrm{Fe}$ & $\mathrm{Fe}$ & 206.4923 & 0.68 & [45] \\
\hline $\mathrm{Fe}$ & $\mathrm{Ti}$ & 398.7154 & 1.0103 & [45] \\
\hline
\end{tabular}

The interatomic potential forms and parameters for $\mathrm{He}-\mathrm{Fe}, \mathrm{He}-\mathrm{Ti}$, and $\mathrm{He}-\mathrm{O}$ interactions were from Juslin and Nordlund [46], Wang et al. [47], and Govers et al. [44], respectively.

At the beginning of the simulation, the energy of the system was minimized with the conjugate gradient algorithm to avoid the overlapping of atoms. Then, the system was allowed to equilibrate using a canonical NVT (constant number of particles, volume and temperature) ensemble at an initial temperature of $280 \mathrm{~K}$. This equilibration period lasted for $0.6 \mathrm{~ns}$ at a timestep of $1 \mathrm{fs}$. Next, the system was heated from the initial temperature to the target temperatures, and the heating process lasted for $0.4 \mathrm{~ns}$. Ten different target temperatures were considered, including $400 \mathrm{~K}, 500 \mathrm{~K}, 600 \mathrm{~K}, 700 \mathrm{~K}, 800 \mathrm{~K}, 900 \mathrm{~K}, 1000 \mathrm{~K}$, $1100 \mathrm{~K}, 1300 \mathrm{~K}$, and $1500 \mathrm{~K}$. The system was maintained at each target temperature for $0.6 \mathrm{~ns}$ and the results were recorded every $5 \mathrm{ps}$.

\subsection{Similitude Analysis}

In an actual lunar environment, the maximum implantation depth of helium-3 ions could reach several hundred nanometers. However, the range of helium-3 trapped in ilmenite under investigation for molecular dynamics simulation was $1 \mathrm{~nm}$. Therefore, similitude analysis is applied in this work to figure out the thermal release characteristics of implanted helium-3 under actual lunar condition. The laws of similarity referring to the physical variables of the model at corresponding points are proportional to its prototype [48]. Thus, combining with scale ratios of the physical quantities and the physical characteristics in the model, the corresponding physical characteristics in its prototype can be obtained.

Determining the basic scale ratios is the key step in the similitude analysis. By selecting the basic scale ratios, the scale ratios of other physical quantities could be derived. Generally, for complete similarity, three basic scale ratios are linear scale ratio $\lambda_{l}$, velocity scale ratio $\lambda_{v}$, and density scale ratio $\lambda_{\rho}$, respectively. However, in this work, considering that the diffusion coefficient is an important factor affecting the thermal release of helium-3, the 
linear scale ratio $\lambda_{l}$, diffusion coefficient scale ratio $\lambda_{D}$, and density scale ratio $\lambda_{\rho}$ are selected as three basic scale ratios. Equations (6)-(8) show the three basic scale ratios:

$$
\begin{aligned}
\lambda_{l} & =\frac{l_{p}}{l_{m}} \\
\lambda_{D} & =\frac{D_{p}}{D_{m}} \\
\lambda_{\rho} & =\frac{\rho_{p}}{\rho_{m}}
\end{aligned}
$$

where $l_{p}, D_{p}$, and $\rho_{p}$ represent length scale, diffusion coefficient scale, and density scale in prototype, respectively; $l_{m}, D_{m}$, and $\rho_{m}$ represent length scale, diffusion coefficient scale, and density scale in model, respectively.

Based on the three basic scale ratios, other scale ratios could be deduced. According to the Einstein relationship, the diffusion coefficient can be calculated as follows [49]:

$$
D=\lim _{t \rightarrow \infty} \frac{1}{6 N_{m} t}\left\langle\sum_{j=1}^{N_{m}}\left(r_{j}(\mathrm{t})-r_{j}(0)\right)^{2}\right\rangle
$$

where $N_{m}$ is the number of particles; $t$ is the time period; $r_{j}(0)$ represents the displacement of particle $j$ at initial time; $r_{j}(\mathrm{t})$ represents the displacement of particle $j$ at time $t ;\langle\ldots\rangle$ represents the ensemble average. Therefore, the time scale ratio can be obtained as

$$
\lambda_{t}=\frac{t_{p}}{t_{m}}=\frac{\lambda_{l}^{2}}{\lambda_{D}}
$$

where $t_{p}$ and $t_{m}$ represent time scales in the prototype and model, respectively.

\subsection{Calculation Process}

The calculation process is shown in Figure 3. First, the implantation of helium-3 ions into the lunar ilmenite was simulated using SRIM code. The corresponding concentration profile of implanted helium-3 ions in ilmenite was obtained. Second, molecular dynamics simulations for thermal release of helium-3 atoms trapped in ilmenite with different concentration profiles were performed to investigate the release characteristics of helium-3. The amounts of released helium-3 were calculated at different temperatures and the optimal heating temperature for helium-3 thermal release was obtained. The diffusion coefficient and activation energy of helium-3 in ilmenite were also analyzed. Finally, the heating time of helium-3 in actual lunar ilmenite was studied using similitude analysis.

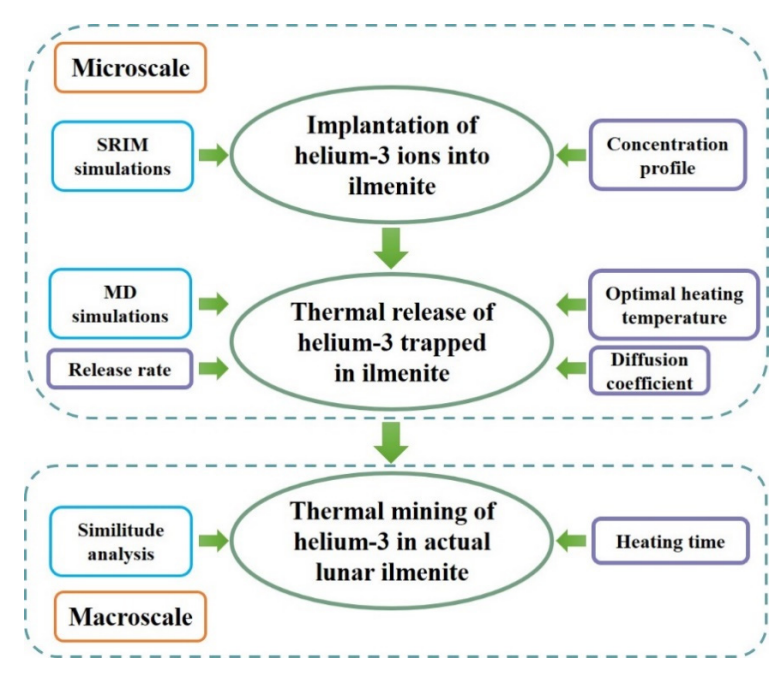

Figure 3. Flow chart of the calculation process. 


\section{Results and Discussion}

\subsection{Validation of Molecular Dynamics Model}

Figure 4 shows the release patterns of helium between molecular dynamics simulations with different concentration profiles and the thermal release experiment in ilmenite from Apollo samples 71,501 [29]. It is seen that the helium release patterns between the thermal release experiment in actual lunar ilmenite and the molecular dynamics calculation results with Gaussian distribution were similar. The release peaks of both curves appeared at about $700 \mathrm{~K}$. The amount of released helium at the peak for the thermal release experiment in actual lunar ilmenite was about $24 \%$, while the amount of released helium at the peak for the molecular dynamics calculation results with Gaussian distribution was about $27 \%$. However, the helium release peak for the molecular dynamics calculation results with uniform distribution appeared at about $500 \mathrm{~K}$. Meanwhile, the corresponding release amount at peak was higher than that for the other two release curves. Thus, the release pattern of helium which was trapped in lunar ilmenite with the concentration profile of Gaussian distribution showed more agreement with the experimental data, compared with that with the concentration profile of uniform distribution.

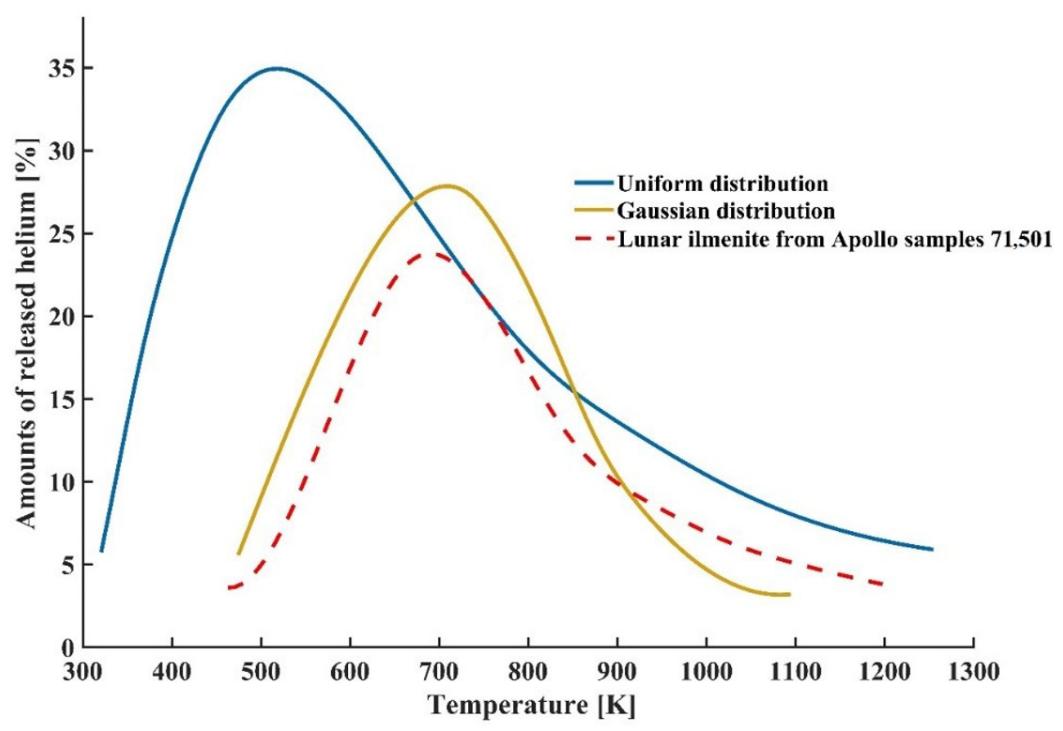

Figure 4. Release patterns of helium between molecular dynamics simulations with different concentration profiles and the thermal release experiment in ilmenite from Apollo samples 71,501 [29].

Table 4 shows the amounts of released helium with different concentration profiles at different temperatures. It is seen that the amounts of released helium with a concentration profile of Gaussian distribution was larger than that with uniform distribution between $300 \mathrm{~K}$ and $700 \mathrm{~K}$. However, the amounts of released helium with a concentration profile of uniform distribution was greater than that with Gaussian distribution at $900 \mathrm{~K}$ and $1100 \mathrm{~K}$. At high temperatures, the helium in deep layers started to release. Since the amount of implanted helium in deeper layers with a concentration profile of uniform distribution was larger than that with Gaussian distribution, the corresponding amount of released helium was larger at relatively high temperatures.

Table 4. Amounts of released helium with different concentration profiles at different temperatures.

\begin{tabular}{cccc}
\hline Temperature(K) & $\begin{array}{c}\text { Amounts of Released Helium with } \\
\text { Uniform Distribution (\%) }\end{array}$ & $\begin{array}{c}\text { Amounts of Released Helium with } \\
\text { Gaussian Distribution (\%) }\end{array}$ & $\begin{array}{c}\text { Differences between Two } \\
\text { Concentration Profiles }\end{array}$ \\
\hline 300 & 0 & 16 & 16 \\
500 & 35.4 & 40 & 4.6 \\
700 & 57.5 & 62 & 4.5 \\
900 & 73 & 70 & -3 \\
1100 & 82 & 75 & -7 \\
\hline
\end{tabular}




\subsection{Determination of Optimal Heating Temperature}

Figure 5 shows the variations in cumulative amounts of released helium- 3 with the temperature. It is seen that the cumulative amounts of released helium-3 increased rapidly at first and then tended to stabilize. When the temperature was relatively low, the increase in temperature mainly caused the helium-3 located in the shallow layer of the ilmenite to diffuse rapidly to the surface and then be released. When the temperature was relatively high, the helium-3 in the deep layer of the ilmenite also began to be released. In addition, compared with the helium-3 atoms in vacancies, the helium-3 atoms located in interstitials had a smaller binding energy, which means that the interstitial helium- 3 atoms were more unstable than the helium-3 atoms in lattice vacancies. Therefore, the helium- 3 atoms in interstitials were easier to release at lower temperature. It is seen from Figure 5 that when the temperature was $700 \mathrm{~K}$, most of interstitial helium-3 atoms were released. When the temperature was greater than $700 \mathrm{~K}$, the helium-3 atoms located in vacancies were released due to the large energy of helium-3 at this time. However, compared with the release of helium-3 at low temperatures, the cumulative amounts of released helium- 3 with vacancies as main trapping sites at high temperatures slowed down.

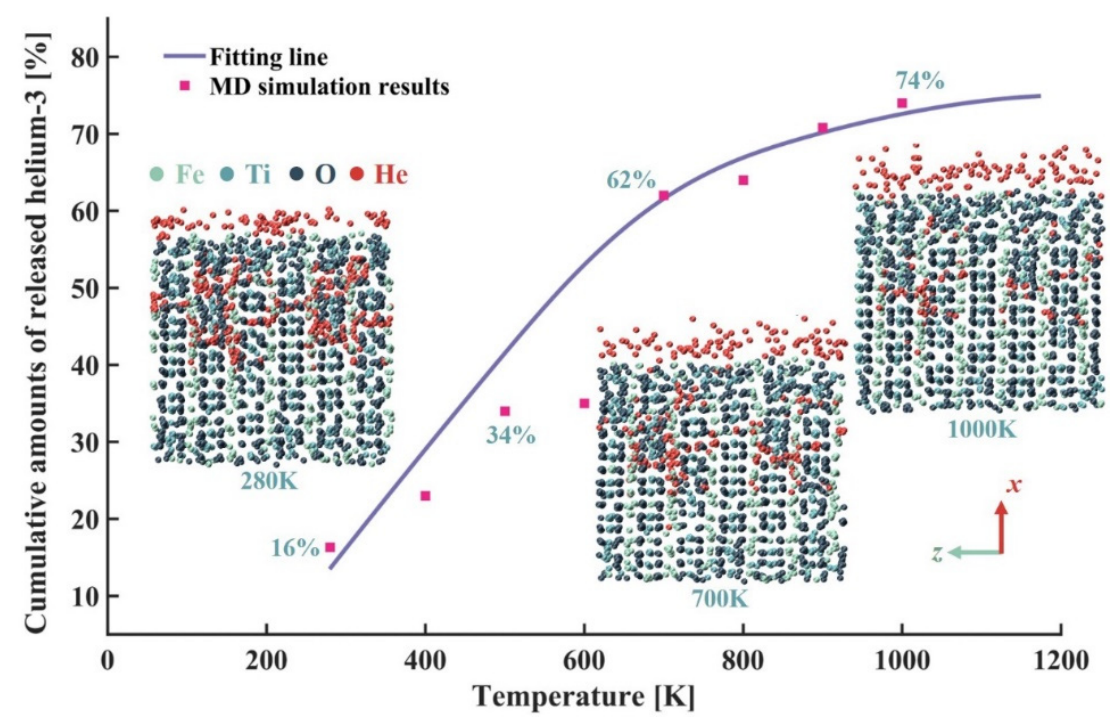

Figure 5. Variations in cumulative amounts of released helium-3 with temperature. The insets show the diffusion and thermal release of helium-3 in ilmenite at $280 \mathrm{~K}, 700 \mathrm{~K}$, and $1000 \mathrm{~K}$, respectively.

It is defined that when the change rate of cumulative amount of released helium-3 $N_{r e}$ with respect to the temperature was not greater than $2 \times 10^{-4} \mathrm{~K}^{-1}$, the cumulative amount of released helium-3 no longer increased with the temperature, that is,

$$
\frac{d N_{r e}}{d T} \leq 2 \times 10^{-4}
$$

At this time, the calculated temperature was $1000 \mathrm{~K}$. Therefore, the optimal heating temperature of helium-3 should be set at $1000 \mathrm{~K}$ and the corresponding cumulative amount of released helium-3 was about $74 \%$.

\subsection{Diffusion Coefficient and Activation Energy of Helium-3 in Lunar Ilmenite}

Figure 6 shows the variations in diffusion coefficient of helium-3 in ilmenite with temperature. It is seen that as the temperature increased, the diffusion coefficient of helium-3 increased exponentially. According to the Arrhenius relationship, there is [50]

$$
D(T)=\frac{D_{0}}{a^{2}} \cdot e^{-\frac{E_{a}}{R T}}
$$


where $D$ is the diffusion coefficient, $D_{0}$ is the frequency factor, $E_{a}$ is the activation energy for diffusion, $a$ is the characteristic diffusion length, $R$ is the gas constant and $T$ is the absolute temperature.

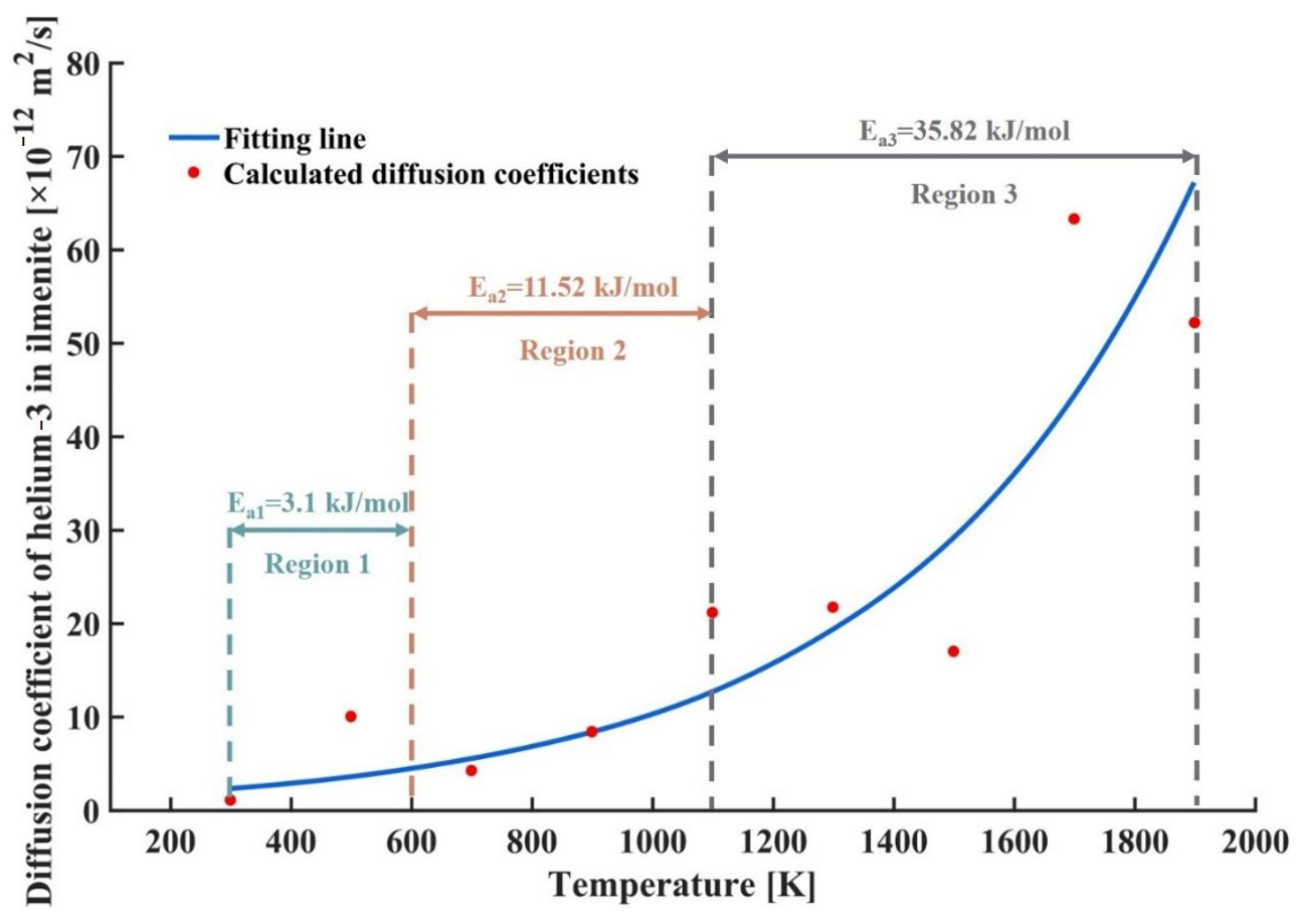

Figure 6. Variations in diffusion coefficient of helium-3 in ilmenite with temperature.

Taking logarithm on both sides of Equation (12), Equation (12) is transformed into

$$
\ln D=\ln \frac{D_{0}}{a^{2}}-\frac{E_{a}}{R T}
$$

If $\frac{1}{T}$ is selected as the independent variable and $\ln D$ is selected as the dependent variable, the slope represents $-\frac{E_{a}}{R}$, and the activation energies for diffusion of helium-3 at different temperatures can be calculated. Three average activation energies were obtained for region 1 (temperature range from $300 \mathrm{~K}$ to $600 \mathrm{~K}$ ), region 2 (temperature range from $600 \mathrm{~K}$ to $1100 \mathrm{~K}$ ), and region 3 (temperature range from $1100 \mathrm{~K}$ to $1900 \mathrm{~K}$ ), respectively, as shown in Figure 6. It is seen that as the temperature rose, and the activation energy for diffusion of helium-3 also increased, indicating that more energy was supplied to helium-3 atoms for the diffusion.

\subsection{Release Rate of Helium-3 in Lunar Ilmenite}

Figure 7 shows the variations in cumulative amounts of released helium- 3 from ilmenite with the heating time at different target temperatures. It is seen that in the temperature rise stage (temperature rise from initial temperature of $280 \mathrm{~K}$ to target heating temperatures), the cumulative amounts of released helium-3 increased rapidly. When the temperatures reached the target heating temperatures in the constant temperature stage, the increase of heating time could not further increase the cumulative amounts of released helium-3. It indicates that on the microscale, helium-3 was released rapidly, and the equilibrium for thermal release of helium-3 in ilmenite was reached within $0.4 \mathrm{~ns}$. The helium- 3 atoms trapped in ilmenite were bound by the binding energy of the ilmenite lattice. When the energy of helium-3 was higher than the binding energy of the lattice, helium-3 could be released. The binding energy was related to the forms and implantation depth of helium-3 in ilmenite. The deeper implantation depth of helium-3 led to the easier capture for helium-3 by lattice vacancies during the process of helium-3 diffusion to the 
surface for thermal release. In addition, compared with the interstitial helium-3 atoms, the helium-3 atoms in lattice vacancies were more stable, resulting in more difficult release of helium-3. Therefore, on the microscopic scale, compared with the binding energy, the heating time contributes little to the release of helium-3, which is consistent with Kuhlman's finding [51].

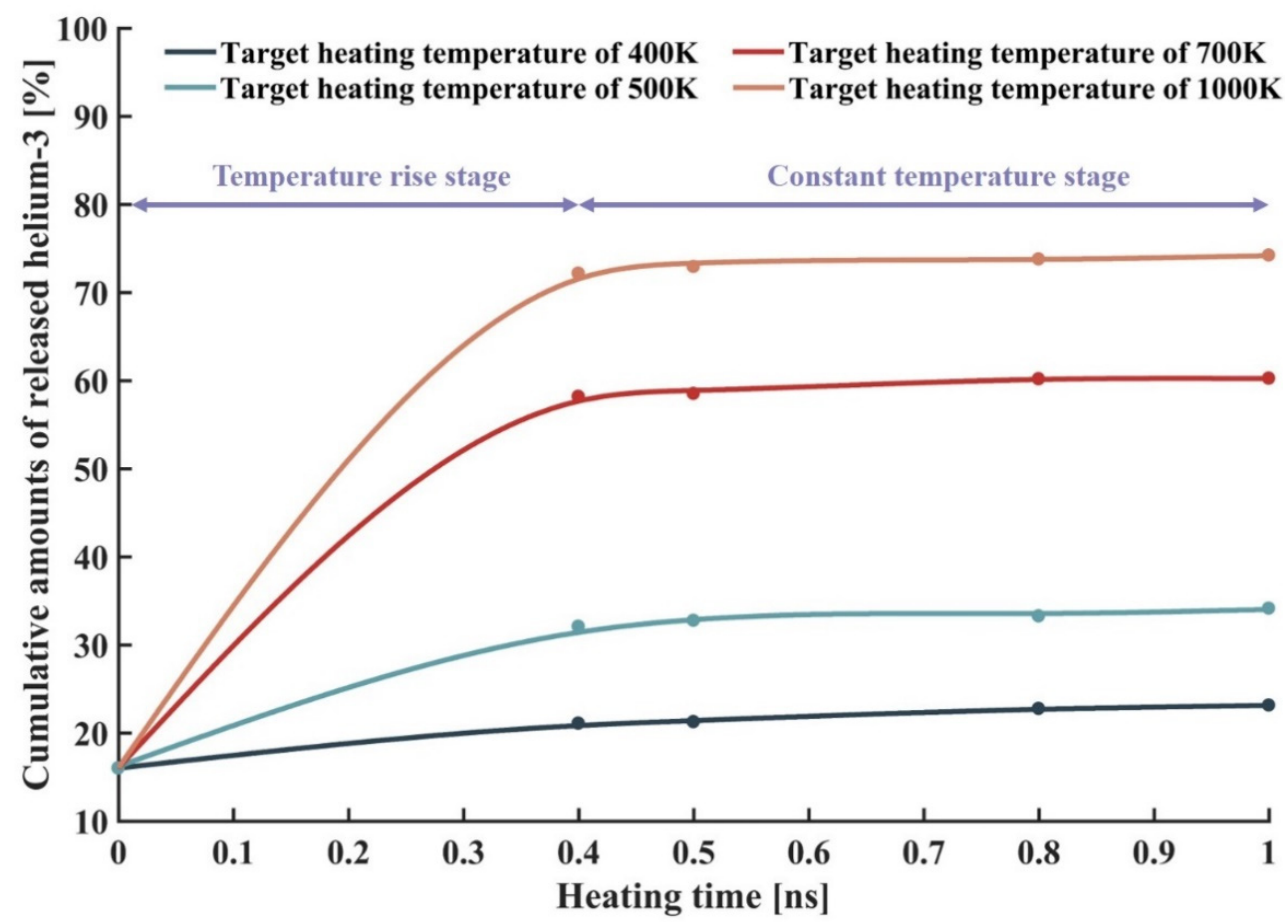

Figure 7. Variations in cumulative amounts of released helium-3 from the ilmenite with heating time at different target temperatures.

\subsection{Heating Time under Actual Lunar Condition}

Table 5 shows the scale ratios at different temperatures for similitude analysis. The calculation of the diffusion coefficient scale ratio was based on Equation (7), and the actual diffusion coefficients of helium-3 were from Fu et al.'s experiments [40]. The linear scale ratio was calculated according to Equation (6). Based on the concentration profile of helium3 via the SRIM in Section 2, the actual implantation depth of helium-3 into ilmenite was taken as $423 \mathrm{~nm}$. According to Equation (10), the time scale ratio was calculated. It is seen from Table 5 that, in general, as the temperature increased, the time scale ratio decreased.

Table 5. Scale ratios at different temperatures for similitude analysis.

\begin{tabular}{ccccccc}
\hline Temperature (K) & $\boldsymbol{D}_{\boldsymbol{m}}\left(\mathbf{c m}^{2} / \mathbf{s}\right)$ & $\boldsymbol{D}_{\boldsymbol{p}}\left(\mathrm{cm}^{2} \mathbf{s}\right)[\mathbf{4 0}]$ & $\lambda_{\boldsymbol{D}}$ & $\lambda_{\boldsymbol{l}}$ & $\lambda_{t}$ & $\boldsymbol{t}_{\boldsymbol{p}}(\mathbf{s})$ \\
\hline 500 & $3.63 \times 10^{-8}$ & $7.00 \times 10^{-15}$ & $1.93 \times 10^{-7}$ & & $9.28 \times 10^{11}$ & 371.13 \\
700 & $5.61 \times 10^{-8}$ & $3.75 \times 10^{-13}$ & $6.69 \times 10^{-6}$ & & $2.67 \times 10^{10}$ & 10.70 \\
900 & $8.39 \times 10^{-8}$ & $3.43 \times 10^{-12}$ & $4.09 \times 10^{-5}$ & 423 & $4.38 \times 10^{9}$ & 1.75 \\
1100 & $12.75 \times 10^{-8}$ & $1.40 \times 10^{-11}$ & $1.10 \times 10^{-4}$ & & $1.63 \times 10^{9}$ & 0.65 \\
1300 & $19.48 \times 10^{-8}$ & $2.69 \times 10^{-10}$ & $1.38 \times 10^{-3}$ & & $1.30 \times 10^{8}$ & 0.052 \\
1500 & $29.24 \times 10^{-8}$ & $3.88 \times 10^{-10}$ & $1.33 \times 10^{-3}$ & & $1.35 \times 10^{8}$ & 0.054 \\
\hline
\end{tabular}

Figure 8 shows the variations in actual heating time for helium-3 trapped in lunar ilmenite with temperature. It is seen that as the heating temperature rose, the actual heating time for thermal release of helium-3 decreased. When the temperature was equal to $700 \mathrm{~K}$, the required heating time was about $11 \mathrm{~s}$. When the temperature was equal to $900 \mathrm{~K}$, the required heating time was less than $2 \mathrm{~s}$. It indicates that for hundreds of nanometers of 
implantation depth of helium-3 in actual lunar ilmenite, when the mining temperature is greater than $700 \mathrm{~K}$, it takes a short time to release the helium-3. For the optimal heating temperature of $1000 \mathrm{~K}$, the thermal release time of helium- 3 is about $1 \mathrm{~s}$.

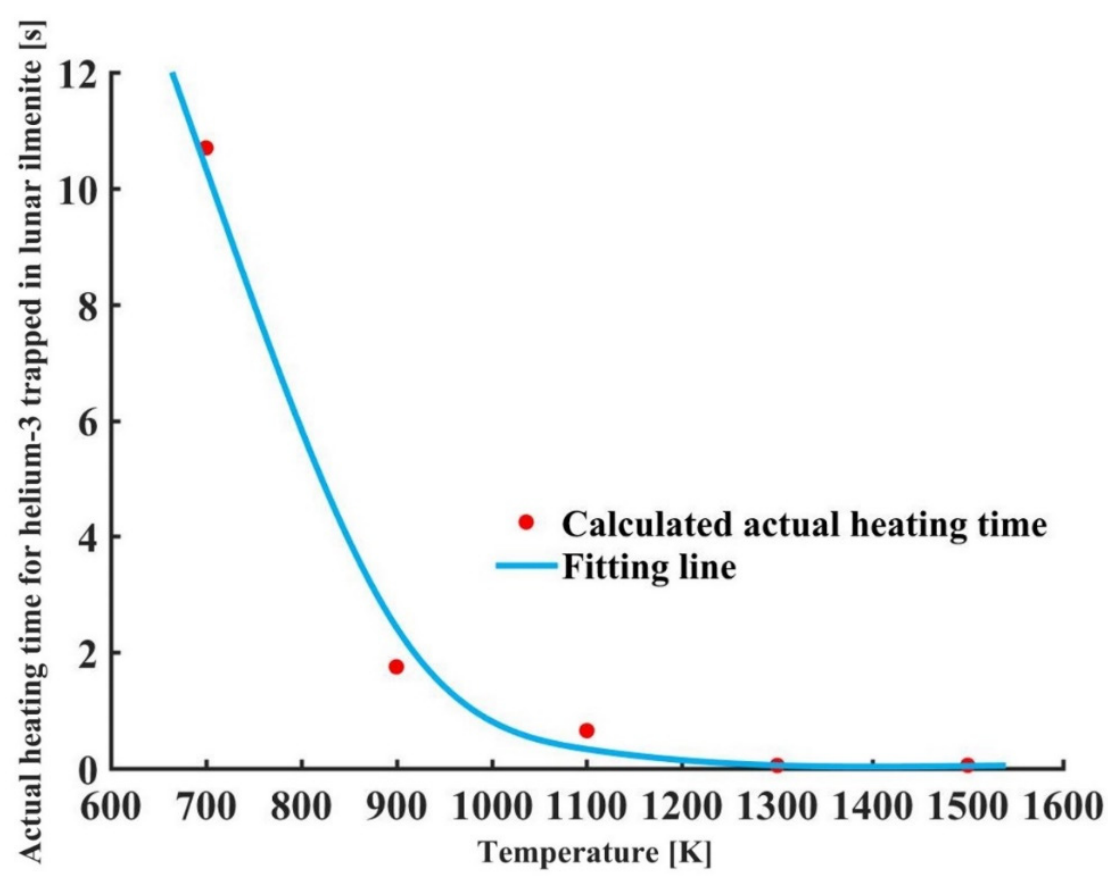

Figure 8. Variations in actual heating time for helium-3 trapped in lunar ilmenite with temperature.

\section{Conclusions}

Investigating the concentration profile of implanted helium-3 in lunar ilmenite and its thermal release mechanism is of great significance for in-situ resource utilization on the Moon. First, the implantation of helium-3 ions into the lunar ilmenite was simulated and the concentration profile of implanted helium- 3 ions in ilmenite was obtained. Then, based on the obtained concentration profile, the thermal release model of helium-3 in ilmenite was established and the thermal release process of helium- 3 on the microscale was investigated via molecular dynamics simulations. The optimal heating temperature, the diffusion coefficient, and the release rate of helium-3 were analyzed. Finally, combining with similitude analysis, the heating time of helium-3 in lunar ilmenite under actual lunar conditions was studied.

The results show that the concentration profile of implanted helium-3 ions in ilmenite followed a Gaussian distribution, and the helium-3 mainly existed in the interstitials and vacancies of the ilmenite crystal. In addition, as the heating temperature increased, the cumulative amounts of released helium- 3 increased rapidly at first and then tended to stabilize. The optimal heating temperature of helium-3 was about $1000 \mathrm{~K}$ and the corresponding cumulative amount of released helium-3 was about $74 \%$. The diffusion coefficient and activation energy of helium-3 increased with the temperature, indicating easier diffusion for helium-3 in ilmenite. When the energy of helium-3 was higher than the binding energy of the ilmenite lattice, helium-3 could be released rapidly on the microscale. Furthermore, the increase of the heating temperature led to the decrease of heating time for thermal release of helium-3 under actual lunar conditions. For the optimal heating temperature of $1000 \mathrm{~K}$, the thermal release time of helium-3 was about $1 \mathrm{~s}$.

For future work, the stability and thermal release ability of helium-3 at lower temperatures would be investigated in order to evaluate the mining potential of helium-3 in permanently shadowed regions at lunar poles. 
Author Contributions: Conceptualization, H.S. and Y.S.; methodology, Y.L.; software, J.Z.; validation, X.Z., J.Z. and J.K.; formal analysis, H.S.; investigation, D.M.; resources, H.S.; data curation, D.M.; writing-original draft preparation, H.S.; writing-review and editing, Y.S.; visualization, D.M.; supervision, H.S.; project administration, H.S.; funding acquisition, H.S. All authors have read and agreed to the published version of the manuscript.

Funding: This research was funded by the Fundamental Research Funds for the Central Universities of China (Grant No. FRF-IC-19-012) and the National Natural Science Foundation of China (Grant No. 11972073).

Institutional Review Board Statement: Not applicable.

Informed Consent Statement: Not applicable.

Data Availability Statement: The data presented in this study are available in this article.

Acknowledgments: The authors would like to thank the reviewers and editors whose critical comments were very helpful in preparing this article.

Conflicts of Interest: The authors declare no conflict of interest.

\section{References}

1. Anand, M.; Crawford, I.A.; Balat-Pichelin, M.; Abanades, S.; van Westrenen, W.; Péraudeau, G.; Jaumann, R.; Seboldt, W. A brief review of chemical and mineralogical resources on the Moon and likely initial in situ resource utilization (ISRU) applications. Planet. Space Sci. 2012, 74, 42-48. [CrossRef]

2. Rasera, J.N.; Cilliers, J.J.; Lamamy, J.A.; Hadler, K. The beneficiation of lunar regolith for space resource utilisation: A review. Planet. Space Sci. 2020, 186, 104879. [CrossRef]

3. Gou, S.; Di, K.; Yue, Z.; Liu, Z.; He, Z.; Xu, R.; Liu, B.; Peng, M.; Wan, W.; Wang, Y.; et al. Forsteritic olivine and magnesium-rich orthopyroxene materials measured by Chang'e-4 rover. Icarus 2020, 345, 113776. [CrossRef]

4. Cesaretti, G.; Dini, E.; De Kestelier, X.; Colla, V.; Pambaguian, L. Building components for an outpost on the Lunar soil by means of a novel 3D printing technology. Acta Astronaut. 2014, 93, 430-450. [CrossRef]

5. Gladstone, G.R.; Hurley, D.M.; Retherford, K.D.; Feldman, P.D.; Pryor, W.R.; Chaufray, J.-Y.; Versteeg, M.; Greathouse, T.K.; Steffl, A.J.; Throop, H.; et al. LRO-LAMP Observations of the LCROSS Impact Plume. Science 2010, 330, 472-476. [CrossRef]

6. Li, S.; Lucey, P.G.; Milliken, R.E.; Hayne, P.O.; Fisher, E.; Williams, J.-P.; Hurley, D.M.; Elphic, R.C. Direct evidence of surface exposed water ice in the lunar polar regions. Proc. Natl. Acad. Sci. USA 2018, 115, 8907-8912. [CrossRef] [PubMed]

7. Sefton-Nash, E.; Williams, J.-P.; Greenhagen, B.T.; Warren, T.J.; Bandfield, J.L.; Aye, K.-M.; Leader, F.; Siegler, M.A.; Hayne, P.O.; Bowles, N.; et al. Evidence for ultra-cold traps and surface water ice in the lunar south polar crater Amundsen. Icarus 2019, 332, 1-13. [CrossRef]

8. Honniball, C.I.; Lucey, P.G.; Li, S.; Shenoy, S.; Orlando, T.M.; Hibbitts, C.A.; Hurley, D.M.; Farrell, W.M. Molecular water detected on the sunlit Moon by SOFIA. Nat. Astron. 2021, 5, 121-127. [CrossRef]

9. Farrell, W.M.; Hurley, D.M.; Esposito, V.J.; McLain, J.L.; Zimmerman, M.I. The statistical mechanics of solar wind hydroxylation at the Moon, within lunar magnetic anomalies, and at Phobos. J. Geophys. Res. Planet. 2017, 122, 269-289. [CrossRef]

10. Farrell, W.M.; Hurley, D.M.; Zimmerman, M.I. Solar wind implantation into lunar regolith: Hydrogen retention in a surface with defects. Icarus 2015, 255, 116-126. [CrossRef]

11. Grumpe, A.; Wöhler, C.; Berezhnoy, A.A.; Shevchenko, V.V. Time-of-day-dependent behavior of surficial lunar hydroxyl/water: Observations and modeling. Icarus 2019, 321, 486-507. [CrossRef]

12. Sowers, G.F.; Dreyer, C.B. Ice Mining in Lunar Permanently Shadowed Regions. New Space 2019, 7, 235-244. [CrossRef]

13. Schwandt, C.; Hamilton, J.A.; Fray, D.J.; Crawford, I.A. The production of oxygen and metal from lunar regolith. Planet. Space Sci. 2012, 74, 49-56. [CrossRef]

14. Sakurai, M.; Sone, Y.; Nishida, T.; Matsushima, H.; Fukunaka, Y. Fundamental study of water electrolysis for life support system in space. Electrochim. Acta 2013, 100, 350-357. [CrossRef]

15. Fa, W.; Jin, Y.-Q. Quantitative estimation of helium-3 spatial distribution in the lunar regolith layer. Icarus 2007, 190, 15-23. [CrossRef]

16. Simko, T.; Gray, M. Lunar Helium-3 Fuel for Nuclear Fusion: Technology, Economics, and Resources. World Futures Rev. 2014, 6, 158-171. [CrossRef]

17. Johnson, J.R.; Swindle, T.D.; Lucey, P.G. Estimated solar wind-implanted helium-3 distribution on the Moon. Geophys. Res. Lett. 1999, 26, 385-388. [CrossRef]

18. Slyuta, E.; Yakovlev, O.; Voropaev, S.; Dubrovskii, A. He implantation and concentrations in minerals and lunar regolith particles. Geochem. Int. 2013, 51, 959-967. [CrossRef]

19. Curran, N.M.; Nottingham, M.; Alexander, L.; Crawford, I.A.; Füri, E.; Joy, K.H. A database of noble gases in lunar samples in preparation for mass spectrometry on the Moon. Planet. Space Sci. 2020, 182, 104823. [CrossRef] 
20. Kim, K.J.; Wöhler, C.; Berezhnoy, A.A.; Bhatt, M.; Grumpe, A. Prospective 3He-rich landing sites on the Moon. Planet. Space Sci. 2019, 177, 104686. [CrossRef]

21. Cocks, F.H. 3He in permanently shadowed lunar polar surfaces. Icarus 2010, 206, 778-779. [CrossRef]

22. Surkov, Y.; Shkuratov, Y.; Kaydash, V.; Korokhin, V.; Videen, G. Lunar ilmenite content as assessed by improved Chandrayaan-1 M3 data. Icarus 2020, 341, 113661. [CrossRef]

23. Shukla, S.; Tolpekin, V.; Kumar, S.; Stein, A. Investigating the Retention of Solar Wind Implanted Helium-3 on the Moon from the Analysis of Multi-Wavelength Remote Sensing Data. Remote Sens. 2020, 12, 3350. [CrossRef]

24. Shkuratov, Y.G.; Starukhina, L.; Kaidash, V.; Bondarenko, N. 3He Distribution over the Lunar Visible Hemisphere. Solar Syst. Res. 1999, 33, 409.

25. Pepin, R.; Nyquist, L.; Phinney, D.; Black, D. Rare gases in Apollo 11 lunar material. Geochim. Cosmochim. Ac. Supplement 1970, 1, 1435.

26. Srinivasan, B.; Hennecke, E.; Sinclair, D.; Manuel, O. A comparison of noble gases released from lunar fines (\#15601.64) with noble gases in meteorites and in the earth. Lunar Planet. Sci. Conf. Proc. 1972, 2, 1927-1945.

27. Baur, H.; Frick, U.; Funk, H.; Schultz, L.; Signer, P. Thermal release of helium, neon, and argon from lunar fines and minerals. Lunar Planet. Sci. Conf. Proc. 1972, 2, 1947-1966.

28. Hohenberg, C.; Davis, P.; Kaiser, W.; Lewis, R.; Reynolds, J. Trapped and cosmogenic rare gases from stepwise heating of Apollo 11 samples. Geochim. Cosmochim. Acta 1970, 1, 1283.

29. Pepin, R.O.; Becker, R.H.; Schlutter, D.J. Irradiation records in regolith materials. I: Isotopic compositions of solar-wind neon and argon in single lunar mineral grains. Geochim. Cosmochim. Acta 1999, 63, 2145-2162. [CrossRef]

30. Burgess, K.D.; Stroud, R.M. Phase-dependent space weathering effects and spectroscopic identification of retained helium in a lunar soil grain. Geochim. Cosmochim. Acta 2018, 224, 64-79. [CrossRef]

31. Anufriev, G.S. Hopping diffusion of helium isotopes from samples of lunar soil. Phys. Solid State 2010, 52, 2058-2062. [CrossRef]

32. Harris-Kuhlman, K.R. Trapping and Diffusion of Helium in Lunar Minerals. Ph.D. Thesis, University of Wisconsin-Madison, Madison, WI, USA, 1998.

33. Futagami, T.; Ozima, M.; Nagal, S.; Aoki, Y. Experiments on thermal release of implanted noble gases from minerals and their implications for noble gases in lunar soil grains. Geochim. Cosmochim. Acta 1993, 57, 3177-3194. [CrossRef]

34. Mueller, H.; Jordan, J.; Kalbitzer, S.; Kiko, J.; Kirsten, T. Rare gas ion probe analysis of helium profiles in individual lunar soil particles. Lunar Planet. Sci. Conf. Proc. 1976, 7, 937-951.

35. Wieler, R.; Baur, H.; Signer, P. Noble gases from solar energetic particles revealed by closed system stepwise etching of lunar soil minerals. Geochim. Cosmochim. Acta 1986, 50, 1997-2017. [CrossRef]

36. Ziegler, J.F.; Ziegler, M.D.; Biersack, J.P. SRIM-The stopping and range of ions in matter (2010). Nucl. Instrum. Meth. B 2010, 268, 1818-1823. [CrossRef]

37. Saha, U.; Devan, K.; Ganesan, S. A study to compute integrated dpa for neutron and ion irradiation environments using SRIM-2013. J. Nucl. Mater. 2018, 503, 30-41. [CrossRef]

38. Loeffler, M.J.; Dukes, C.A.; Baragiola, R.A. Irradiation of olivine by $4 \mathrm{keV} \mathrm{He+:} \mathrm{Simulation} \mathrm{of} \mathrm{space} \mathrm{weathering} \mathrm{by} \mathrm{the} \mathrm{solar} \mathrm{wind.}$ J. Geophys. Res. Planet. 2009, 114, 1-13. [CrossRef]

39. Mueller, H.; Kiko, J.; Kirsten, T. High resolution depth profiles of rare gases in individual lunar soil particles. Lunar Planet. Sci. Conf. Proc. 1976, 7, 577-579.

40. Fu, X.; Zou, Y.; Zheng, Y.; He, H.; Ouyang, Z. Noble gas diffusion mechanism in lunar soil simulant grains: Results from 4He+ implantation and extraction experiments. J. Earth Sci. China 2011, 22, 566. [CrossRef]

41. Fan, H.; Chen, D.; Liu, P.; Duan, H.; Huang, Y.; Long, M.; Liu, T. Structural and transport properties of FeO-TiO $\mathrm{F}_{2}$ system through molecular dynamics simulations. J. Non Cryst. Solids 2018, 493, 57-64. [CrossRef]

42. Belashchenko, D.K.; Ostrovski, O.I.; Skvortsov, L.V. Molecular dynamics simulation of binary $\mathrm{CaO}-\mathrm{FeO}, \mathrm{MgO}_{-} \mathrm{SiO}{ }_{2}, \mathrm{FeO}_{-} \mathrm{SiO}$, $\mathrm{CaO}-\mathrm{SiO}_{2}$ and ternary $\mathrm{CaO}-\mathrm{FeO}-\mathrm{SiO}_{2}$ systems. Thermochim. Acta 2001, 372, 153-163. [CrossRef]

43. Zhang, J.; Song, H.; Zhu, W.; Wang, J. Liquid Transport through Nanoscale Porous Media with Strong Wettability. Transport Porous Med. 2021, 1-15. [CrossRef]

44. Govers, K.; Lemehov, S.; Hou, M.; Verwerft, M. Molecular dynamics simulation of helium and oxygen diffusion in $\mathrm{UO}_{2 \pm \mathrm{x}}$. J. Nucl. Mater. 2009, 395, 131-139. [CrossRef]

45. Rappe, A.K.; Casewit, C.J.; Colwell, K.S.; Goddard, W.A.; Skiff, W.M. UFF, a full periodic table force field for molecular mechanics and molecular dynamics simulations. J. Am. Chem. Soc. 1992, 114, 10024-10035. [CrossRef]

46. Juslin, N.; Nordlund, K. Pair potential for Fe-He. J. Nucl. Mater. 2008, 382, 143-146. [CrossRef]

47. Wang, J.; Hou, Q.; Sun, T.Y.; Wu, Z.C.; Long, X.G.; Wu, X.C.; Luo, S.Z. Simulation of Helium Behaviour in Titanium Crystals Using Molecular Dynamics. Chinese Phys. Lett. 2006, 23, 1666-1669. [CrossRef]

48. Jin, J.; Ling, Y.; Hao, Y. Similarity analysis of parabolic-trough solar collectors. Appl. Energ. 2017, 204, 958-965. [CrossRef]

49. Wang, S.; Javadpour, F.; Feng, Q. Molecular dynamics simulations of oil transport through inorganic nanopores in shale. Fuel 2016, 171, 74-86. [CrossRef]

50. Watson, E.B.; Baxter, E.F. Diffusion in solid-Earth systems. Earth Planet. Sc. Lett. 2007, 253, 307-327. [CrossRef]

51. Kuhlman, K.R.; Kulcinski, G.L. Helium Isotopes in the Lunar Regolith-Measuring Helium Isotope Diffusivity in Lunar Analogs. Moon Springer 2012, 23-56. [CrossRef] 